ACCEPTED MANUSCRIPT

\title{
Non-intrusive measurements of free-water-surface profiles and fluctuations of turbulent, two-phase flow using 2-D laser scanner
}

To cite this article before publication: Gašper Rak et al 2020 Meas. Sci. Technol. in press https://doi.org/10.1088/1361-6501/ab727f

\section{Manuscript version: Accepted Manuscript}

Accepted Manuscript is "the version of the article accepted for publication including all changes made as a result of the peer review process, and which may also include the addition to the article by IOP Publishing of a header, an article ID, a cover sheet and/or an 'Accepted Manuscript' watermark, but excluding any other editing, typesetting or other changes made by IOP Publishing and/or its licensors"

This Accepted Manuscript is @ 2020 IOP Publishing Ltd.

During the embargo period (the 12 month period from the publication of the Version of Record of this article), the Accepted Manuscript is fully protected by copyright and cannot be reused or reposted elsewhere.

As the Version of Record of this article is going to be / has been published on a subscription basis, this Accepted Manuscript is available for reuse under a CC BY-NC-ND 3.0 licence after the 12 month embargo period.

After the embargo period, everyone is permitted to use copy and redistribute this article for non-commercial purposes only, provided that they adhere to all the terms of the licence https://creativecommons.org/licences/by-nc-nd/3.0

Although reasonable endeavours have been taken to obtain all necessary permissions from third parties to include their copyrighted content within this article, their full citation and copyright line may not be present in this Accepted Manuscript version. Before using any content from this article, please refer to the Version of Record on IOPscience once published for full citation and copyright details, as permissions will likely be required. All third party content is fully copyright protected, unless specifically stated otherwise in the figure caption in the Version of Record.

View the article online for updates and enhancements. 


\title{
Non-intrusive measurements of free-water- surface profiles and fluctuations of turbulent, two-phase flow using 2-D laser scanner
}

\author{
Gašper Rak ${ }^{1}$, Marko Hočevar ${ }^{2}$ and Franci Steinman ${ }^{1}$ \\ ${ }^{1}$ Chair of Fluid Mechanics, Faculty for Civil and Geodetic Engineering, University of Ljubljana, \\ Ljubljana, Slovenia \\ ${ }^{2}$ Laboratory for Water Turbine Machinery, Faculty of Mechanical Engineering, University of Ljubljana, \\ Ljubljana, Slovenia
}

E-mail: gasper.rak@fgg.uni-lj.si (corresponding author)

Received xxxxxx

Accepted for publication $\mathrm{xxxxxx}$

Published xxxxxx

\begin{abstract}
For measuring free-water surfaces conventional methods, such as resistance-type probes, Umanometers, point gauges, ultrasonic sensors, etc. are still most commonly used in modern hydro engineering. These methods give accurate results at suitable flow conditions, but are insufficient when the water surface is characterized by turbulence and two-phase flow, with fast dynamics. This paper presents the use of laser scanning as a measurement method for the acquisition of free-water-surface profiles of hydraulic phenomena with turbulent, nonstationary, and non-homogeneous free-surface flows. Results shows that laser scanning can provide accurate measurements of free-water-surface profiles with high spatial and temporal resolution, even in cases of turbulent flows with high vertical fluctuations on the water surface. The comparison with the reference values determined by analysing the images taken with the high-speed camera showed that measurement uncertainty ranges from $\pm 5 \mathrm{~mm}$ to \pm 10 $\mathrm{mm}$, which is more than an order of magnitude smaller than the range of local vertical watersurface fluctuations. The average profiles, envelopes of vertical water surface fluctuation around the mean value, as well as the construction of complex water surface topography of intensive waving can be determined by processing laser scanning data. The results contribute to the wider use of this non-contact measurement method, which provides important information on water flow properties to many fields of hydro-engineering. The application of 2-D laser scanning for free-water-surface acquisition was conducted in the model of supercritical junction flow, where the development of standing waves leads to the phenomenon of self-aerated flow.
\end{abstract}

Keywords: laser scanning; turbulent, two-phase flow; water surface topography; fluctuations

\section{Introduction}

Knowledge of hydraulic phenomena is well advanced mainly in subcritical flows but is still limited in supercritical flows and transitions from one to two or multi-phase flow [1, 2]. According to our overview of expected future challenges in design of hydraulic structures, the main research topics are expected to focus on complex hydraulic phenomena in terms of high-speed flow and particularly air flow features, like 
high-speed outlets, hydraulic power plant flow fields, sediment bypass tunnels, desilting and fish migration facilities, drop shafts etc. [2]. Insufficient or inadequate knowledge of such phenomena often leads to underdimensioning of structures, their poor functionality, and poor operation, while accompanying processes, which also impair the functioning and condition of hydraulic structures and arrangements, are not sufficiently considered. To obtain new information and knowledge about phenomena and processes, experimental, numerical, or even hybrid research is of great importance. An overview of the research shows that due to the complexity of the water flow structures of demanding hydraulic phenomena, there is a lack of quality data for calibration and verification of numerical models, which means that 3D numerical modelling does not yet yield completely satisfactory results [3-5]. According to the mentioned problem, measurements are an important part of hydraulic engineering and it is crucial to take precise and reliable measurements of hydraulic phenomena. For velocity measurements the development of non-intrusive measurement methods, particularly image processing techniques like particle image velocimetry [6], bubble image velocimetry [7], optical flow [8], and computer-aided visualization methods based on the advection-diffusion equation $[9,10]$, has been significant in recent years. More advanced image processing techniques also enable velocimetry of wavy aerated flows (micro-PIV) [11] and bubbly pipe flows [12], visualization and estimation of thickness distribution of vertical air-water annular flow (planar laser-induced fluorescence) [13-16]. Such strong development is not the case in measurements of water levels, where conventional methods, such as resistancetype and pressure probes, ultrasonic sensors, etc. still prevail. These methods give accurate results at suitable flow conditions, but fail to facilitate the measurement of complex, fast time-varying, free-water-surface profiles of aerated flows. Aerated flows have been studied experimentally, numerically, and theoretically over the last few decades and air/water flow characteristics can described in terms of length and time scales, turbulent fluctuations, turbulent kinetic energy, dissipation rate, shear stresses, void fraction, bubble frequencies, and velocities [17]. While a void fraction of an aerated flow is often measured with various electric or optical sensors [18], the most commonly used devices for measuring water levels and depths conventional methods are still, for example, U manometers [19], point gauges [20, 21], and ultrasonic sensors [22, 23]. Zang et al. [23] provide an up-todate literature review on point water level measurement methods and their capabilities, as well as limitations in several types of turbulent flows. The performance of these measurement methods is limited for two-phase flows, because they do not allow for automated continuous measurement of the water surface profile with high temporal and spatial resolution. Due to these issues, research focuses in aerated air- water surface measurements are now shifting to high-speed video cameras in side-view perspective, and to laser-based technologies. Among these, the laser triangulation method performed by fast camera imaging may be used for aerated turbulent water surface height measurement as shown in [24]. The method may be used in a laboratory with a continuous light sheet or in combination with laser scanner illumination for more localized measurements.

This paper presents the applicability of laser scanning for measuring the free-water surface and fluctuations of turbulent, high-aerated open channel flows with strong/vertical and longitudinal dynamics. The main focus of this ștudy is on postprocessing of laser scanning point cloud, and on determining the remission value threshold and range of water surface fluctuation. Analysis was performed using the remission data of each scan. Point clouds of water surface measurements are laden with water height fluctuations, several consecutive specular laser beam reflections, variations of foam layer thickness and properties etc. We will show that the use of remission data improves watér surface measurement results by a large margin, by effectively reducing the scatter of laser scanner measurements. The robustness and versatility of laser scanning has been shown in different fields of research and engineering [25-27]. The laser beam reflects on the still water surface at the same angle as the incident angle or penetrates through the water and reflects from bottom, bubbles or suspended particle. Smith et al. [28] and Tamari et al. [29] studied influence of refraction angle and large incidence angle, respectively, on measured water levels. Despite this, laser scanning has rarely been used for water surface profile measurements. Think tanks from Blenkinsopp and Allis have applied the method for laboratory profile measurements of the time-varying free-water surface of propagating waves [3031]. Both groups have performed measurements in wave flumes and have used water mixed with particulate matter to improve reflection. High frequency measurements of wave transformation and characteristics using a LIDAR scanner were also performed in the field [32-35]. In recent years laser scanning has been proven to be a reliable measuring method for acquiring the free-surface profiles of aerated, turbulent flows [36]. In laboratory studies such laser scanning was efficiently used to record mean standing waves topography [37]. For the supercritical junction flow, standing wavetopography mesh models were analysed for 168 scenarios, from which phenomenological models among independent geometric and hydraulic flow parameters and the dependent characteristics of flow structures were derived [38]. Laser scanning also provided free-surface features in fully aerated flows down a laboratory stepped spillway at high spatial and temporal resolution [36]. Montano et al. [39, 40] used LIDAR measurements to simultaneously record jump toe oscillations and free-surface fluctuations. This enabled new insights into 
the interactions of jump toe and free-surface features in aerated hydraulic jumps.

Due to the specular reflection of the laser beam on the surface of the clear water, the reflected signal reaches the laser scanning sensor only at a null angle (or very close to $0^{\circ}\left( \pm 1^{\circ}\right)$ ). If the beam is reflected off the water's surface away from the laser scanner at a greater angle, the sensor could only receive a signal after several reflections from various surfaces. In such a scenario, the traveling time and consequently the measured distance would be too long and erroneously excessive.

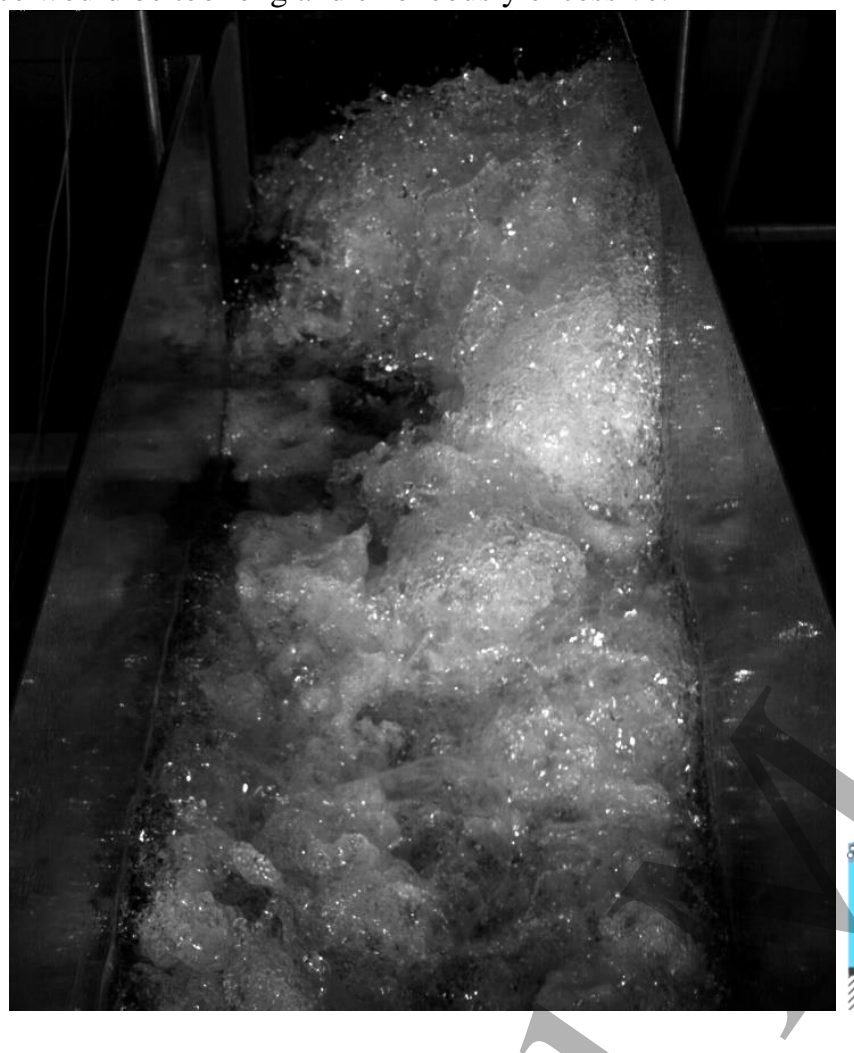

In our study we applied laser scanning to measure freewater-surface profiles accompanied by complex hydraulic phenomena with highly aerated flow and non-stationary behaviour. As an interesting practical case we selected a supercritical junction flow, where distinctively 3D, turbulent, two-phase flow occurs (Figure 1, left). The main aim of our study was to measure transversal profile of the water surface and the range of water surface fluctuation.

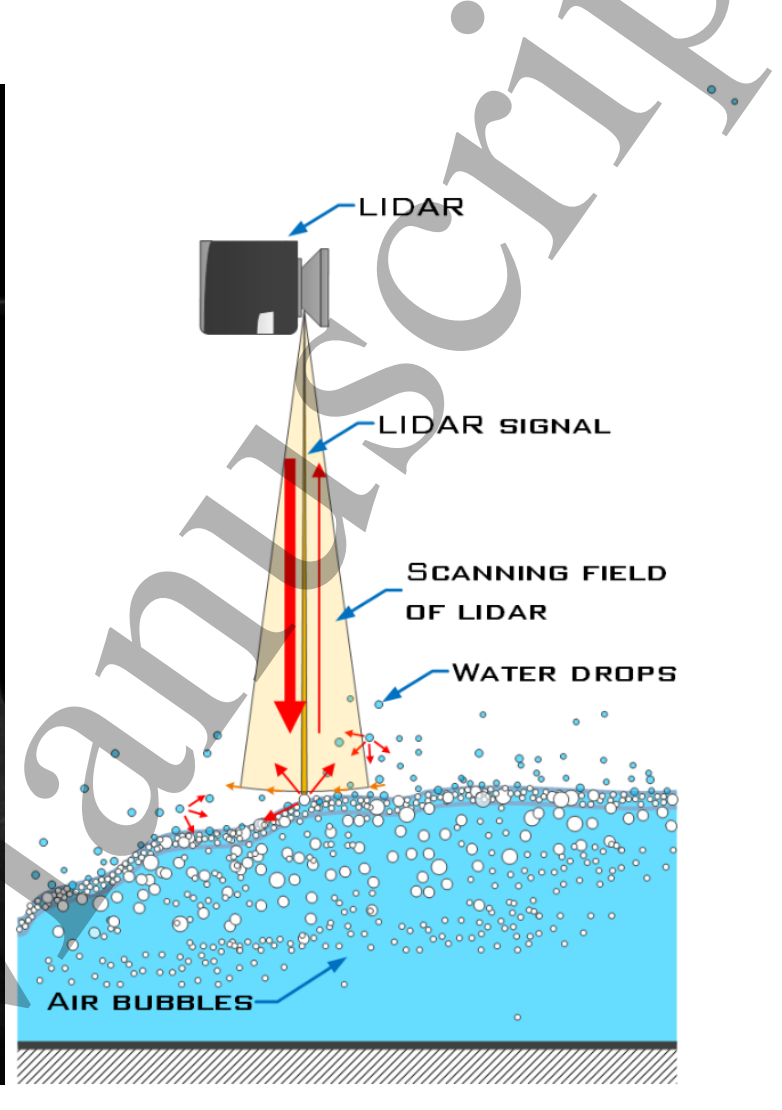

Figure 1. Highly turbulent and aerated supercritical junction flow (left) and a sketch of specular reflections of the laser scanner beam on the aerated water surface (right).

In hydraulic phenomena with highly developed two-phase flow, especially where white water (foaming), spraying, and transitions between different types of aerated flows (from stratified and bubbly to dispersed flow) are present, the exact detection and determination of the water surface is very difficult and challenging. In addition, distinctive fluctuations of water surface or the upper layer of the mixture of water and air are also frequently present. An abundance of droplets above the surface can lead to overestimates of surface height, numerous steep waves prevent measurements of concave surfaces, entrapped bubbles below the surface can underestimate surface height, and the presence of foam can increase measurement uncertainty (Figure. 1, right). But at the same time such flow properties create conditions under which, unlike for other measurement methods, laser scanning can give useful results.

\section{Experimental set-up}

\subsection{Supercritical junction flow model}

The experimental work was done at the hydraulic laboratory of the Faculty of Civil and Geodetic Engineering of the University of Ljubljana. A junction model with $90^{\circ}$ angle between the main channel and its side channel axes was used. The lengths of incoming flows with supercritical flow were the same for the main and tributary channels, i.e. $1 \mathrm{~m}$, to ensure equal upstream conditions at the start of the junction. The length of the main channel downstream of the junction was $4.5 \mathrm{~m}$. The channel walls were made entirely from glass panels with joints between them kept to a minimum, so that the impact of roughness on flow conditions was also minimized. The inflows into the channels from the reservoir 
were separated from each other with two pipelines equipped each with a valve and an electromagnetic flowmeter. The inflows were provided with pressure vessels, where both pressure vessels allowed for the adjustment of the opening and thus of the desired flow conditions. The experimental apparatus was equipped with a frame structure for mounting and precise positioning of measuring devices that allowed for the repeatability of measurements in the same cross-sections. The frame structure was separately mounted on a rigid metal supporting base to prevent the transmission of vibrations from the glass part to the measuring equipment. The model is shown in Figure 2, where the main parts are marked with numbers: 1 - pipeline system equipped with valves and flowmeter; 2 pressure vessels with adjustable height of the openings; 3 main channel; 4 - tributary channel; 5 - rails with carrier for mounting and precise positioning of measurement devices; 6 - laser scanner; 7 - free overflow at the end of the model.

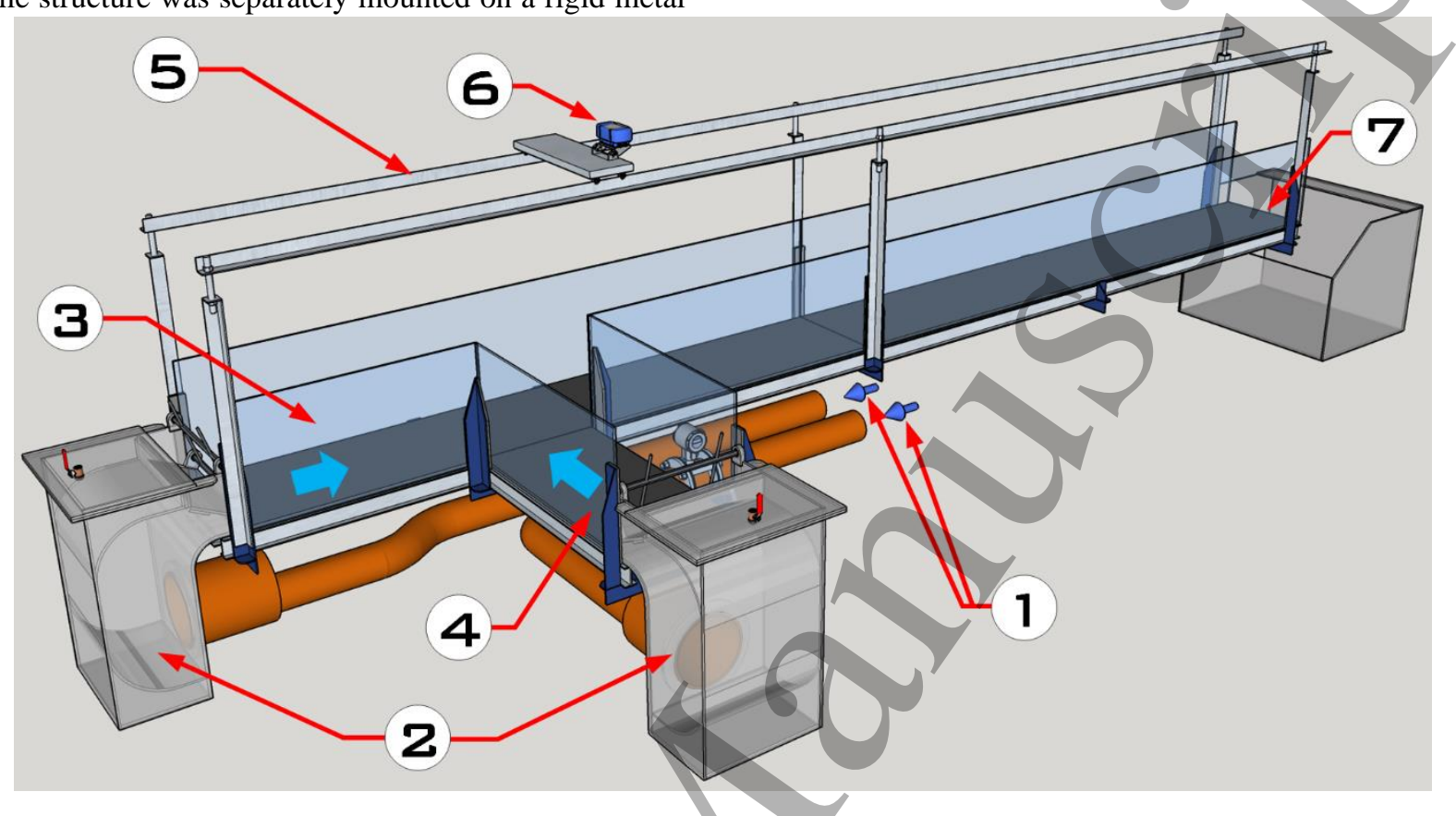

Figure 2. Experimental setup - supercritical junction flow model.

By adjusting the height of the openings and discharge in each pipeline we set flow parameters for the series of scenarios. The discharge in the main and tributary channel was set from 2 to 60 1/s and 1.5 to $55 \mathrm{l} / \mathrm{s}$, respectively. For both channels the opening heights were set at an interval from 10 to $30 \mathrm{~mm}$. The maximum values of the Froude and Reynolds number of the inflows in the analysed scenarios were 12 and $1.2 * 10^{5}$, respectively. The paper presents measurement results of scenarios with the opening height of $20 \mathrm{~mm}$ on both pressure vessels; the discharge in the main channel $37.61 / \mathrm{s}(\mathrm{Fr}$ $=8.5)$, and the discharge in the side channel $251 / \mathrm{s}(\mathrm{Fr}=5.6)$.

\subsection{Measuring equipment and measurements}

The non-stationary topography of free-water-surface flow was measured using a 2-D laser scanner LMS400 manufactured by SICK, which allows for measurements with a high temporal and spatial resolution (Figure 3, left). The device emits laser beams in the visible red light wavelength $\lambda$ $=650 \mathrm{~nm}$ with a frequency up to $175 \mathrm{kHz}$, and the receiver records the reflections from water surfaces, bubbles, walls, etc. Water surface measurements using a laser scanner comprise a relatively new field, so before use for systematically measuring water surface topography the measurement method had to be extensively verified. The verification procedure is described in [37]. Topography measurements along the junction in a length of $2 \mathrm{~m}$ were carried out in 21 consecutive cross-sections at an interval of $100 \mathrm{~mm}$ (Figure 3, right). The results and a comparison with laser triangulation in dashed cross sections (R) and (C1-C3), respectively, are presented later. The laser triangulation method used is described in [24]. Each free-water-surface profile was measured from the centre point transversally to the channel. The angles of the beams' incidence on the water surface were smaller, which increased the number of received, i.e. returned, echoes. The transversal free-water-surface profile in the individual cross-sections was determined based on 6,000 scan lines. Every measurement included distance, angle, and remission data. A configuration with a line scanning frequency $270 \mathrm{~Hz}$ and angular resolution $0.2^{\circ}$ was selected. The angular range was $70^{\circ}$ and each scanline was composed of 350 measurement points; i.e. totalling 94,500 distance measurements per second and 2,100,000 points per cross-section in total. The amount of data recorded is high due to the high scanning frequency required by the non-stationary junction flow phenomena, but the actual acquired data is 
dependent on the reflection from the surface. Often the returned beam is not detected by the laser scanner and data are randomly missing for a range of acquisition angles and times. Despite the high sampling rate and the great number of measurements, only around $5 \%$ of the signal emitted from the laser scanner was, due to the specificity of interaction between laser beam and surface of aerated water, returned and detected by the laser scanner's photodetector.

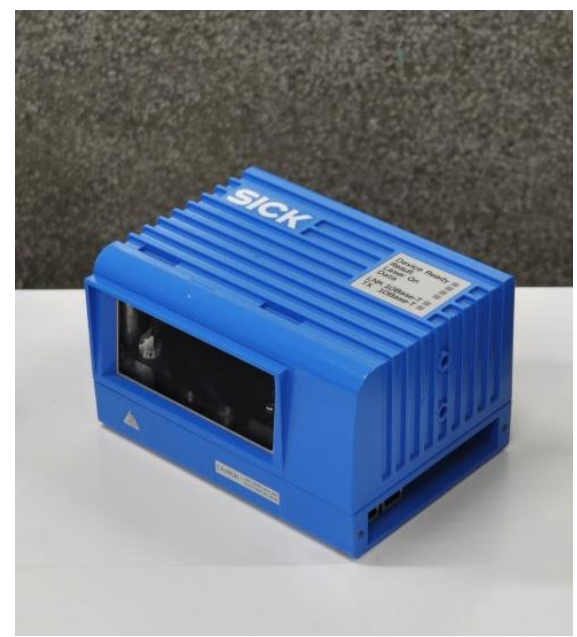

For systematic measurements with a high frequency in many recorded cross-sections, we developed methodology for determining the free-water-surface profile, entire topography of the standing waves, and the envelopes of maximum and minimum values of surface fluctuations. We have developed our own laser scanner drivers for data acquisition, as well as a user interface for configuration, visualization, and data storage.

Figure 3. Laser scanner SICK LMS400 (left) and a sketch of the junction with its main dimensions and measured crosssections (cross-sections in which results are given are marked with red and blue dash lines) (right).

Due to the limitations of conventional measurement methods for measuring the free-water-surface profiles of turbulent, two-phase flow with strong vertical dynamics, reference measurements in selected points were conducted using image analysis. For visualization and to determine the local average water surface level and fluctuations in individual points we used a thin slice ruler inserted into the water flow and a high-speed Casio EX-F1 camera with an image acquisition rate of $300 \mathrm{fps}$. Considering flow conditions, the influence of the ruler on the turbulent two-phase flow could be assumed as negligible. The average value and standard deviation of fluctuation were determined based on the reading of water levels in 1000 consecutive images. In image analysis it was possible to visually control the water levels, as determined by algorithms developed in MATLAB. This also made it possible to assess the fluctuation of the water surface with greater confidence and to compare the values with the results of the analysis of the measurements/with laser scanner.

\subsection{Filtering the measurements based on reflection intensity}

Besides the distance between the device and measuring surface, determined based on the time-of-flight measuring method, the laser scanner also records the remission (intensity) of each returned signal. The remission value is recorded/with a value between 0 and 255 (8-bit record) and doesn't have a measurement unit. Due to the scattering, energy dissipation when traveling through water and reflections on surfaces smaller than the laser beam footprint (only part of the light beam is reflected), the energy of the returned signal is smaller than the energy of the emitted signal from the laser scanner. Figure 4 clearly shows that echoes also come from reflections off of deeply submerged bubbles and water drops above the water's surface. The threshold value for actual water surface measurement filtering was determined by analysing the remission value of the reflections for submerged bubbles in standing water [37]. In this study threshold values were analysed for submerged bubbles and flying droplets of aerated, turbulent flow of the supercritical junction model. By excluding the returned signals with low remission value, we were able to significantly reduce the influence of the bubbles deeper in the water and droplets in the air in determining the mean water surface profile. Multiple returned signals could be detected by the laser scanner due to the scattering of the beam on the turbulent, aerated water surface. The laser scanner used in our study can detect up to three echoes for each emitted signal, but only the first was considered in the post-processing of the raw point cloud. The second and third returned signals frequently originate from the multiple consecutive reflections from various surfaces, which is reflected in the signal's longer 
traveling time, and consequently longer measured distance and apparent lower water depth.

\section{Results and discussion}

\subsection{Average free-water-surface profiles and range of fluctuation measurement}

Figure 4 shows a raw (cyan colour points) and filtered (dark blue colour points) point cloud as measured by laser scanning. In Figures $4 \mathrm{~A}-4 \mathrm{C}$, the raw point cloud was filtered using different values of remission threshold $(\mathrm{A}-$ threshold value $=$ $20 ; \mathrm{B}-$ threshold value $=10 ; \mathrm{C}-$ threshold value $=5$ ). The black dash and the red lines indicate the mean free-watersurface profile, as derived from the entire raw and filtered point cloud, respectively. The black points with error bars show the reference values determined through analysis of the images recorded with a high-speed camera. It is not always evident from the entire point cloud in the individual crosssections where the highest water surface fluctuations occur. The intensity of fluctuations depends on flow characteristics. In areas with a strong transversal dynamic of the water mass, fluctuations are higher closer to the channel walls. In a mixing zone of both supercritical inflows (main and tributary inflows) the highest fluctuations occur along the standing wave ridge inside the channel.

The filtered point cloud has fewer points for high remission thresholds, since reflections that retain much energy are rare (Figure 4, A). High-energy dissipation is a consequence of the laser beam scattering and consecutive specular reflections on the aerated water surface, as well as the beam's traveling through the water itself. With the filtering at a threshold value of 40 and higher, the number of points is too small to determine transversal profile without making further assumptions and running additional analysis. 

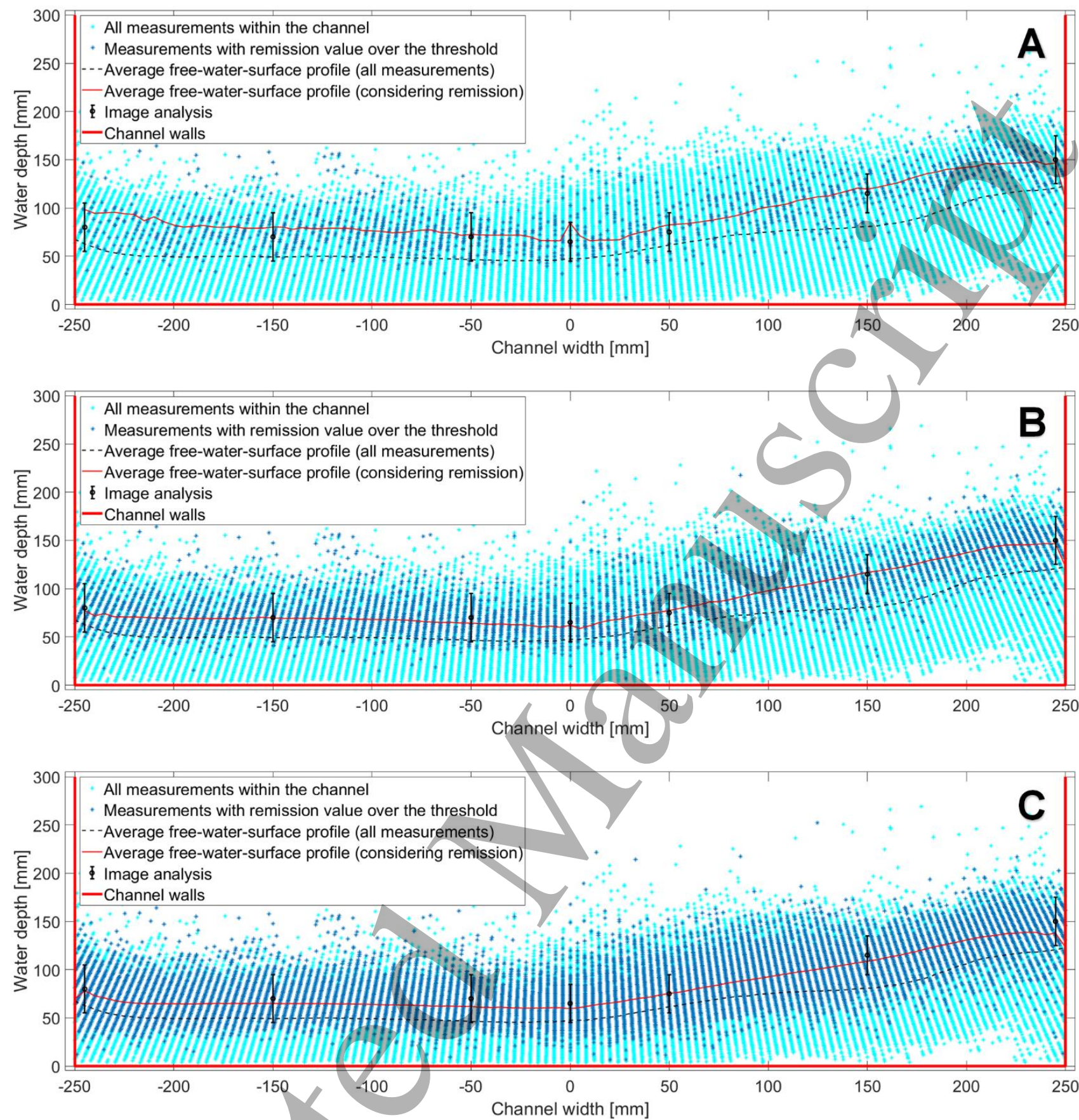

Figure 4. Raw and filtered laser scanning point clouds for remission thresholds 20 (A), 10 (B) and 5 (C); average freewater-surface profiles obtained from raw and filtered point cloud, and reference values obtained with image analysis (cross section "R" on fig. 3, right); depth and Froud numbers of the inflows: $\mathrm{h}_{1}=\mathrm{h}_{2}=20 \mathrm{~mm}, \mathrm{Fr}_{1}=8.5$ and $\mathrm{Fr}_{2}=5.6$ ).

Comparison of both measurement methods shows, that at a lower threshold value in the filtered point cloud there are still many reflections from more deeply immersed bubbles and droplets of spray above the water surface (Figure 4, C). Points with a remission value above the threshold are also located outside the range of the water surface fluctuation, as determined with image analysis. Since the laser beams are reflected on both the drops above the surface and the immersed bubbles below the water surface, the transversal profile still matches the measurements with image analysis.

The measurement showed that laser scanning can be only used to capture the formation of water surfaces where foamed or fully developed two-phase flow occurs in the upper layer. In poorly developed two-phase flow with low bubble density and when bubbles occur across the entire depth of the water body, there are more reflections and a wider range of incidence angles. The determined values are lower than the 
actual ones due to the reflections from the bubbles deeper in the water. The influence of the bubbles deeper in the water could be successfully reduced by filtering the point cloud and taking into account the remission (intensity of reflected signal).

Remission analysis of the returned signals, as detected by the laser scanner's photodetector, shows that it is necessary to find the appropriate value of threshold that enables the determination of the mean free-water-surface profile as well as its fluctuation. In this verification procedure the most appropriate threshold value was determined at 10 . As can be seen from Figure 4.B, the agreement between the mean freewater-surface profile determined from the laser scanning point cloud and the reference levels from image analysis were very good. At the same time, the band of filtered points almost completely coincides with the maximum and minimum envelope of the water surface fluctuations determined through image analysis. The deviations between the laser scanners and the reference measurements are given in Table 1. Based on the measurement uncertainty of the laser scanner provided by the manufacturer and the results presented here, we estimate that after filtering measurements based on the remission data the measurement uncertainty ranges from $\pm 5 \mathrm{~mm}$ for locations with small vertical dynamics to $\pm 10 \mathrm{~mm}$ for locations with high vertical surface dynamics.

Table 1. Comparison of water surface measurements in several points at selected cross-sections using the laser scanner and image analysis with a high-speed camera.

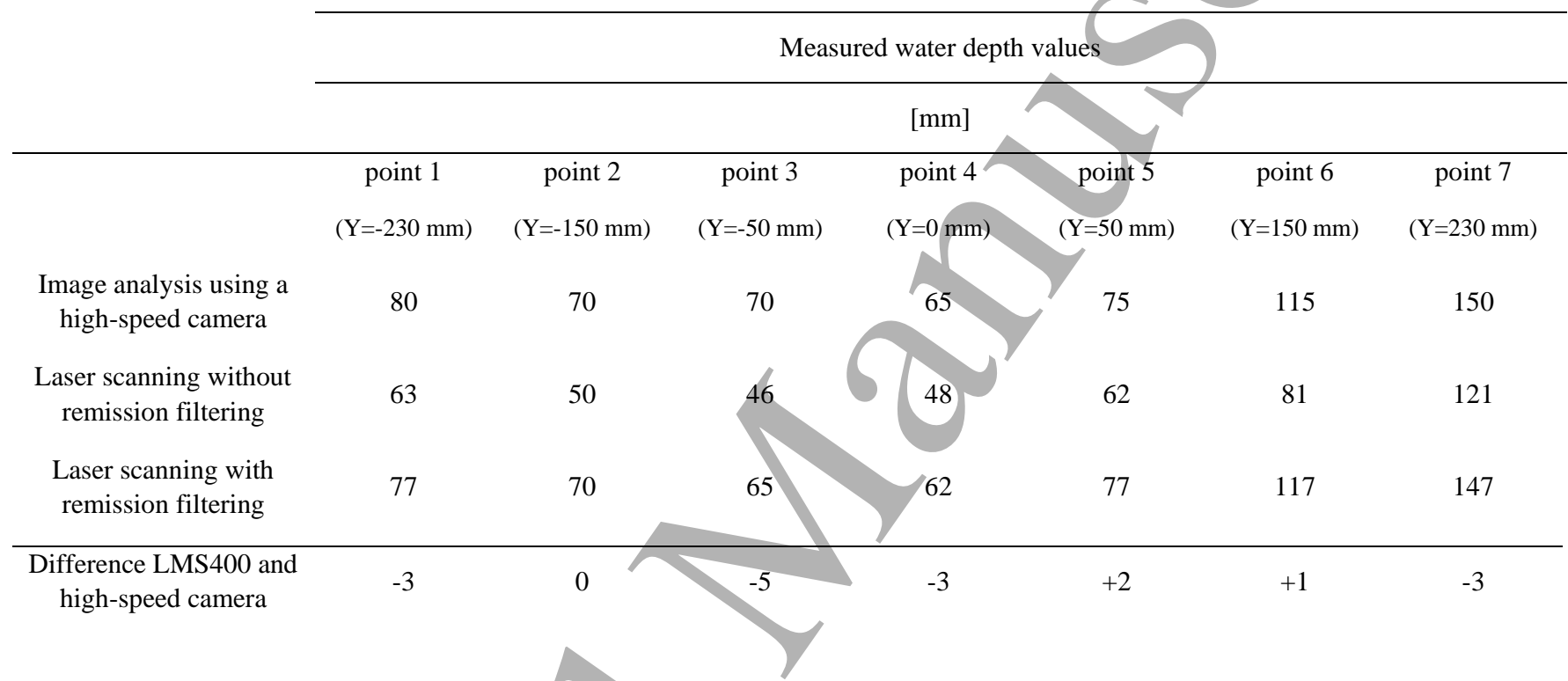

Figure 5 (below) shows histograms of the water depth in three locations as shown in Figure 5 (top), corresponding to locations $\mathrm{x}=-150 \mathrm{~mm}$ (left), $0 \mathrm{~mm}$ (central), and $150 \mathrm{~mm}$ (right location) within the cross-section. Reference measurements with high-speed camera were also taken in these locations. In the analysis all points from the filtered point cloud of 3000 scanlines, located within a $20 \mathrm{~mm}$ wide interval around each $\mathrm{x}$ location were taken into account. 

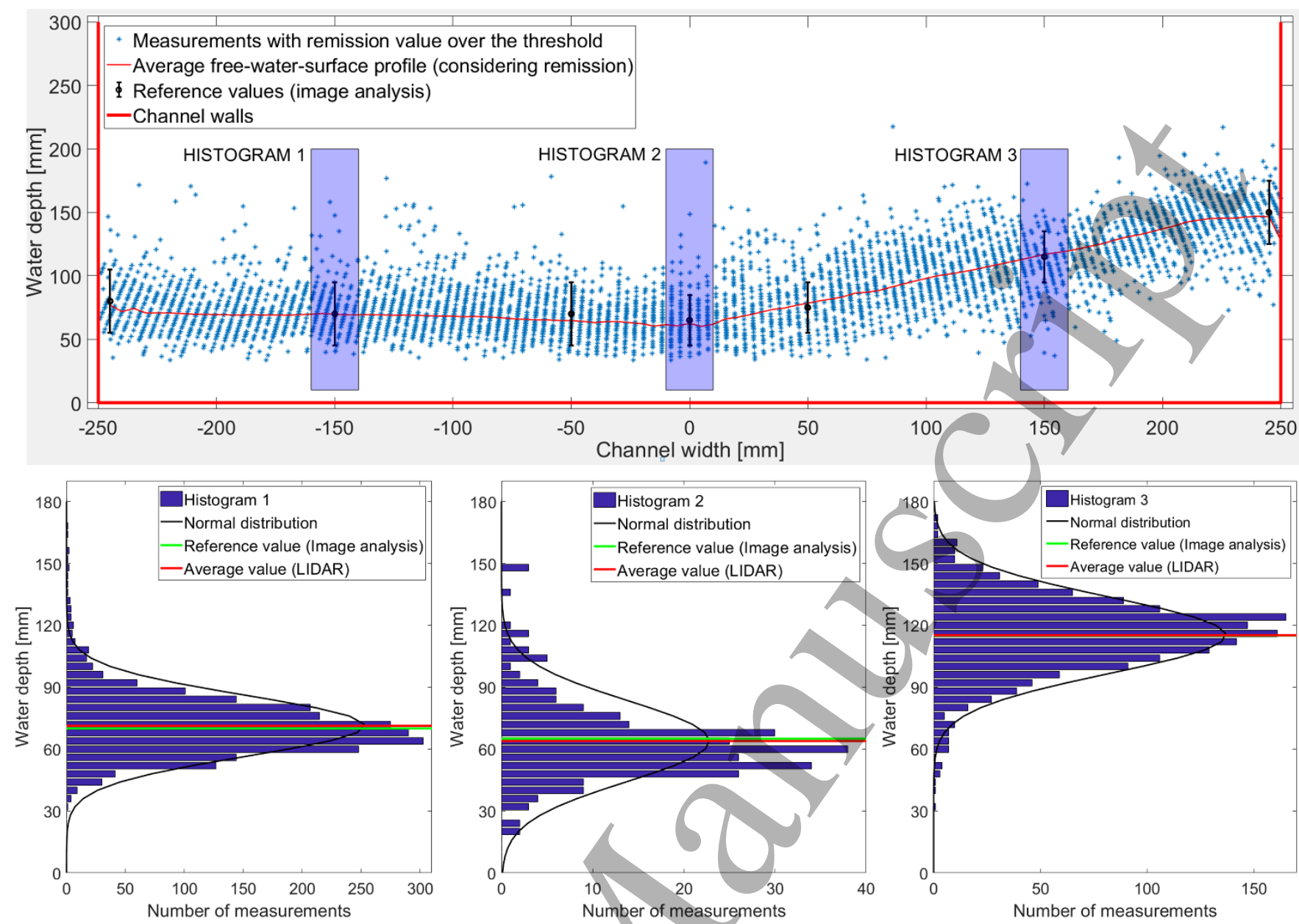

Figure 5. Histograms of water surface measurements in three points transversal to the channel (points from the slots with a width of $20 \mathrm{~mm}$ were taken into account); reference values obtained with image analysis (cross section " $\mathrm{R}$ " in Figure 3, right; depth and Froud numbers of the inflows: $\mathrm{h}_{1}=\mathrm{h}_{2}=20 \mathrm{~mm}, \mathrm{Fr}_{1}=8.5$ and $\mathrm{Fr}_{2}=5.6$ ).

The histograms of water depth values show that the distribution of the measured values approximately corresponds to the normal distributions around a mean water level (determined both by image analysis and laser scanner measurements). In the left slot the measurements are evenly distributed around the mean value, which can be attributed to the turbulent nature of the flow phenomena and strong fluctuation. In the middle slot (evaluation point 2), that was directly below the laser scanner, there is a larger number of measurements in the narrow band around average depth value. At a null angle (or very close to $0^{\circ}$ ) the laser beam penetrates the water slightly better and more deeply than at greater incidence angles. In the right slot distribution is similar to the normal distribution. In all three slots there are a relatively small number of measurements with greater deviation (outliers). Since the point cloud was filtered at a constant value of remission threshold, it was not possible to completely eliminate reflections from more deeply immersed bubbles and droplets above the water surface.
For three cross sections with different vertical dynamics of water surface $\mathrm{C} 1$ to $\mathrm{C} 3$, comparisons of mean free-surface profile and a range of fluctuations obtained by post-processing simultaneous measurements with laser scanning and laser triangulation are shown in Figure 6, left. Flow conditions (dynamics and turbulence) in these cross sections are shown in the images in Figure 6, right. In the post-processing of laser scanning point clouds, the same value was used for the remission threshold as described above (the constant value was set to 10). Comparison shows good agreement (between \pm 10 to $25 \mathrm{~mm}$ ), except for the region near the wall, where the agreement between both methods is slightly worse. Due to the reflection from the glass walls, the performance of the laser triangulation was decreased [24].

The results shown in Figure 6 confirm that remission filtering performed well in representing remarkably different flow conditions (variations in height and velocity fluctuations, surface foam presence and thickness, etc.). As discussed in the Introduction, there is no other method with good temporal and

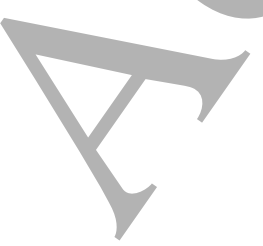


spatial resolution available for benchmarking except for the laser triangulation method (Figure 6).

Good agreement between the results of surface measurements using laser scanner and laser triangulation show that the filtering procedure using remission thresholds
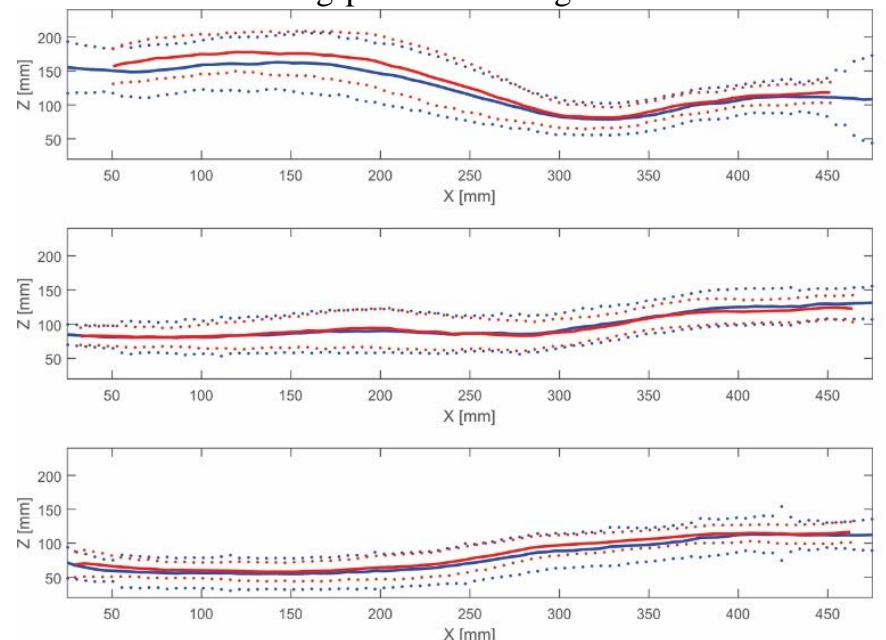

presented above is appropriate. We therefore believe that remission filtering procedure can be successfully used for surface height measurement in a wide range of flow conditions.
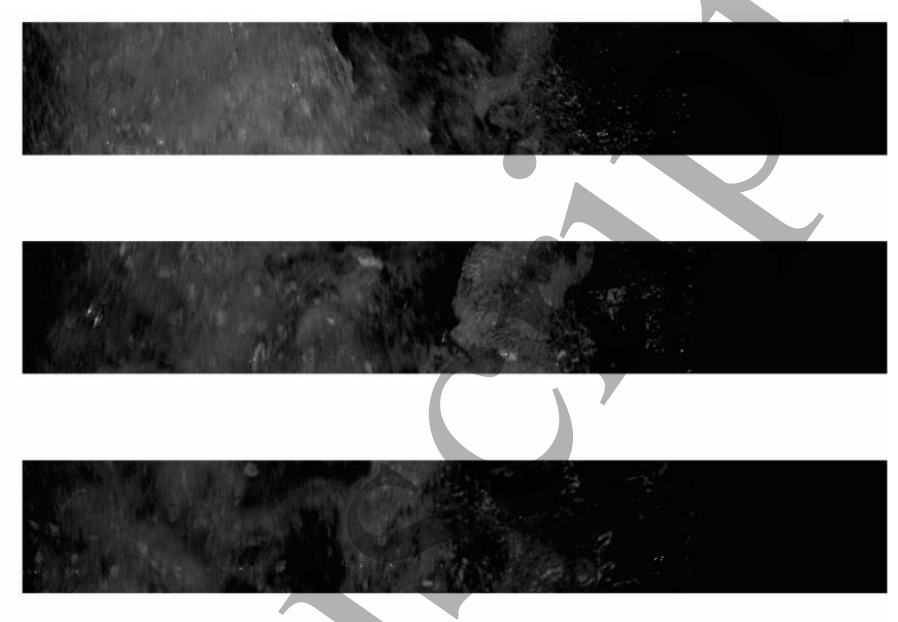

Figure 6. Left - results of measurements of average turbulent water surface profiles (solid lines) and a range of fluctuations (left: dotted lines). Results are shown for laser scanning (blue) and laser triangulation method (red). Right - flow condition in cross sections, where comparison with laser triangulation was made (cross sections "C1-C3" in Figure 3, right; depth and Froud numbers of the inflows: $\mathrm{h}_{1}=\mathrm{h}_{2}=20 \mathrm{~mm}, \mathrm{Fr}_{1}=8.5$ and $\mathrm{Fr}_{2}=5.6$ ).

\subsection{Mesh models of water surface topography and envelopes of fluctuation}

Based upon the methodology of measurement from subsection 3.1, the flow at the entire junction was measured and evaluated. The topography of the standing waves was reconstructed from the free-water-surface profiles of each cross-section (locations shown in Figure 3). The raw point cloud was processed based on the remission values of the laser measurements. Figure 7 (left) shows two consecutive crosssections. We note that the point clouds of the water surface show similar spreading of measured depths and numbers of points in the point cloud. This led to the determination of the mean values of the free-water surface profile, as well as the range of water surface fluctuations. The results are shown in Figure 7 (top, right). The mesh plot was generated using the Kriging method. The remission threshold was set to 10 (Figure $4, \mathrm{~B})$. 


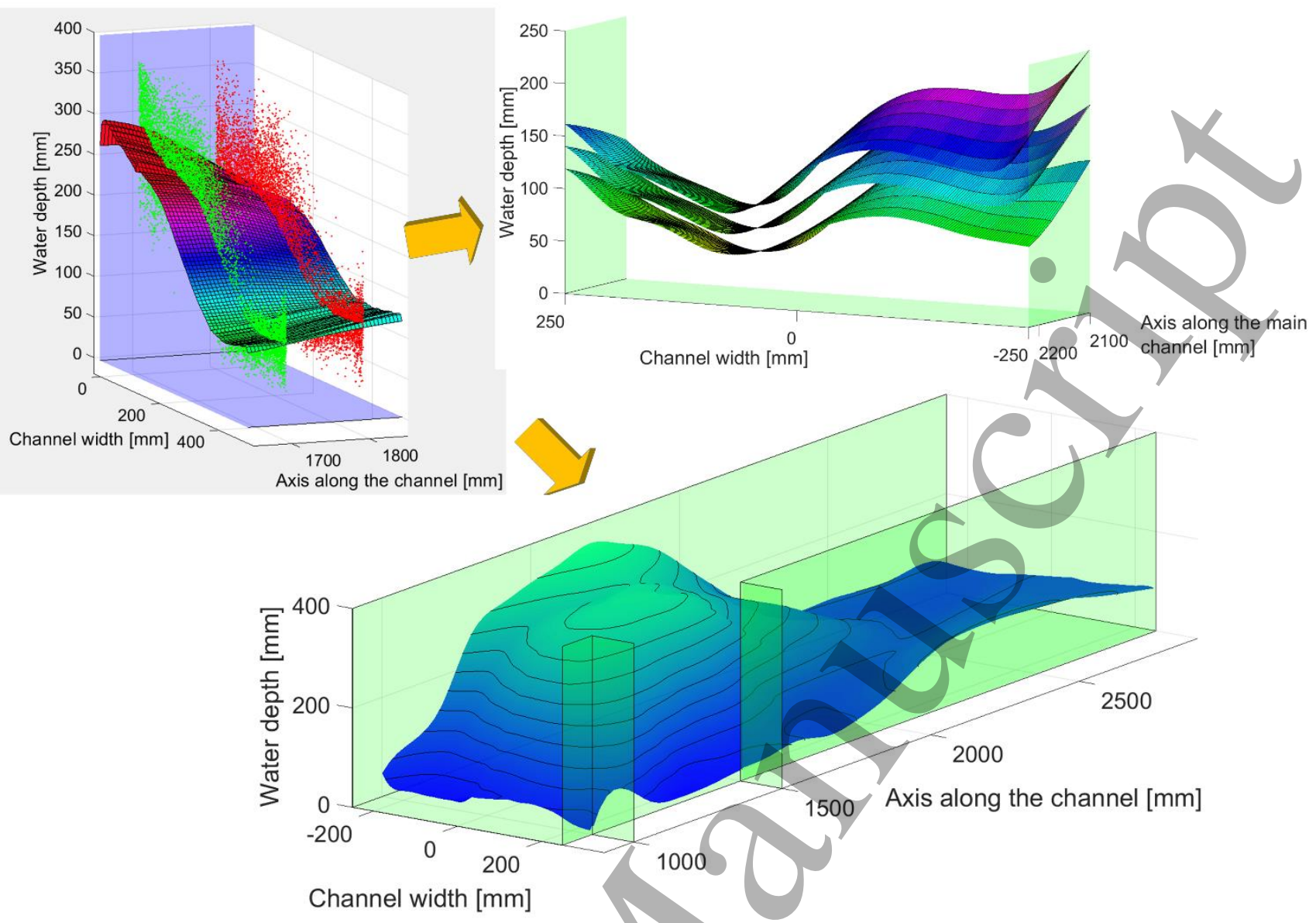

Figure 7. Point clouds of two consecutive cross-sections (left, above); digital water surface model of standing waves along the entire junction (right, below); $100 \mathrm{~mm}$ long section of standing wave with a plot of the average free-water-surface and maximum and minimum envelopes of its fluctuations (right, above) (depth and Froud numbers of the inflows: $h_{1}=h_{2}=20$ $\mathrm{mm}, \mathrm{Fr}_{1}=8.5$ and $\left.\mathrm{Fr}_{2}=5.6\right)$.

The laser scanning direction was from the top down (i.e. plan view). Thus, the measured water surface does not show air pockets or tunnels of air occurring in the body of the wave. Such pockets may appear below the cover ridge of the wave along the circulation zone of both inflows. As seen in Figure 1, a barrel-like water flow formation occurs at the junction due to high Froude numbers of inflows in both the main and tributary channel. Inner barrel roll surfaces cannot be measured from the selected scanning position. Some water structures could be captured by changing the laser scanner position (below the bottom or from the sides), but due to the model's design it would have required significant modifications, which were not possible at this stage. Beside this a minimum required distance between laser scanner emitter/receiver and measured surface (response time/ working range of the laser scanner) has to be assured without any physical barriers. In a side view arrangement a measurement performance could be affected by reflections from glass walls and water drops thereon.
In the areas with lower point density in the point cloud a comparison of Figure 1 and Figure 7 reveals similarities among regions with limited aeration. However, for the presented measurement scenario of high Froude and Reynolds numbers, it was still possible to successfully generate the $3 \mathrm{D}$ mesh model. Remission filtering facilitated the rejection of outliers from reflections from droplets, splashing, or immersed air bubbles. The filtering also enabled determination of the maximum and minimum water surface fluctuation envelopes. Figure 6 (right, bottom) shows the maximum and minimum water surface fluctuation envelope of length $100 \mathrm{~mm}$. Both envelopes were evaluated as \pm one standard deviation from a mean water level. Due to the rejection of points in the point cloud using remission filtering or beam reflections away from the receiver, and particularly because the measurements were taken in the individual sections one after another, an equidistant representation of temporal dynamics in depth or water mass movement across the entire area is not possible. Nevertheless, based on the results of measuring the steady inflow from both channels we

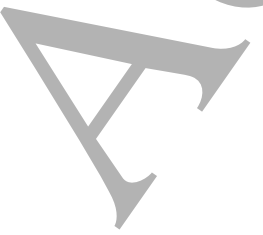


can analyse the extent to which water surface fluctuations occur.

Knowledge of free-water-surface fluctuations in very intense open flow phenomena enables research into and development of hydraulic structures for experimental and applied purpose. The depicted measurement system and analysis procedure of remission filtering proved well suitable for the task. For future work, the vertical dynamics of surfaces in individual locations may be of interest.

In [24, 37], limitations of laser scanning method for measurement of water surface height are well summarized. They originate from the physical properties of several consecutive reflections, in which the beam is reflected back to the laser scanners' receiving optics.

In addition, we would like to address the limitations of the remission filtering method. Results from Figures 4 and 5 show points in a point cloud that are available for remission filtering. Many of them are specularly reflected several times and, with every consecutive reflection, the laser beam's intensity is diminished and thus the remission value smaller. Eventually such measurement becomes invalid, because several reflections apparently increase distance to the objects, which is in this case wrong. Such points are rejected by using remission filtering, increasing measurement accuracy as shown in Figures 4, 5, and 6. But we are left with fewer valid height measurements. Such a procedure may eventually lead to a decrease in measurement resolution or gaps, especially in the case of highly turbulent flows.

There is also an issue with reflections from droplets or bubbles under the surface. Here we may expect a weak first reflection because of the droplet's small size in comparison with the laser scanner's beam diameter. Subsequent reflections from single droplet are much less probable. The remission filtering algorithm will, due to low remission value, recognize the first such reflection from a droplet as a series of reflections and wrongly exclude it from further analysis.

We firmly believe that any future serious development of laser scanning methods for aerated turbulent water surface measurements will use remission filtering to improve measurement accuracy. Recent laser scanner developments, among them those enabling simultaneous velocity measurements, may additionally improve here implemented simple remission filtering method.

\section{Conclusions}

Laser scanning with high spatial and/time resolution is widely used these days. Laser scanning is a non-intrusive measurement method that for a long time was considered unsuitable for measuring water body surfaces. This paper now shows that it is indeed useful as a measurement method for free-water-surface flows with three-dimensional, turbulent, and aerated two-phase flow properties.
Due to the specular laser beam reflections from the water surface, successful measurements (reflected beams detected by laser scanner) in standing, clean water are limited to the incidence angle of the beams at around $0^{\circ}$. In highly aerated flows the continuous air-bubble and water mixture is formed in the surface layer and multiple air/water and water/air surfaces strongly increase the number of specular reflections detected by the laser scanner's photodetector. While the conventional measurement methods typically used in hydro engineering fail to yield satisfactory results in cases of strong two-phase flows with fast water surface dynamics, laser scanning enables acquisition with high temporal resolution and a reconstruction of the high spatial resolution topography. Along with a detailed description of the mean free-watersurface profiles, analysis of raw point clouds makes it possible to determine the water surface fluctuations around the mean values. We were able to filter within the measured point clouds based on remission values. By doing so, returns from flying droplets and submerged bubbles were to a large extent rejected and the water surface scanning was improved. The results extend the use of this measurement method and may provide important information on water flow properties to various fields of hydro-engineering. With filtering and other methods of data post-processing, laser scanning provides a robust solution with applicability in laboratory and field measurements

\section{Acknowledgements}

The authors acknowledge the financial support from the state budget by the Slovenian Research Agency (Programmes P2-0401 Energy engineering and P2-0180 Water Science and Technology, and Geotechnical Engineering: Tools and Methods for Process Analyses and Simulations, and Development of Technologies).

\section{References}

[1] Chanson H 2013 Hydraulics of Aerated Flows: Qui Pro Quo? J. Hydraul. Res. 51 223-243

[2] Hager W H and Boes R M 2014 Hydraulic structures: a positive outlook into the Future J. Hydraul. Res. 52 299-310

[3] Biron P M, Ramamurthy A S and Han S 2004 Threedimensional numerical modeling of mixing at river confluence J. Hydraul. Eng. 130 243-253

[4] Saldarriaga J, Rincon G Moscote G and Trujillo M 2016 Symmetric junction manholes under supercritical flow conditions J. Hydraul. Eng. 130 135-142

[5] Zindovic B, Vojt P, Kapor R and Savic L 2016 Converging stepped spillway flow J. Hydraul. Res. 54 699-707

[6] Amador A, Sánchez-Juny M and Dolz J 2006 Characterization of the nonaerated flow region in a stepped spillway by PIV $J$. Fluid Mech. 128 1266-1273

[7] Rivillas-Ospina G, Pedrozo-Acuna A, Silva R, TorresFreyermuth A and Gutierrez C 2012 Estimation of the velocity 
field induced by plunging breakers in the surf and swash zones Exp. Fluids 52 53-68

[8] Bung D B and Valero D 2014 Optical flow estimation in aerated flows J. Hydraul. Res. 54 575-580

[9] Bizjan B, Orbanić A, Širok B, Kovač B, Bajcar T and Kavler I 2014 A computer-aided visualization method for flow analysis Flow Meas. Instrum. 38 1-8

[10] Müller M, Novak G, Steinman F, Rak G and Bajcar T 2015 Influence of the operating and geometric characteristics of a bottom-hinged flap gate on the discharge coefficient of a side weir J. Mech. Eng. 61 498-506

[11] Schubring D, Foster R E, Rodríguez D J and Shedd T A 2009 Two-zone analysis of wavy two-phase flow using microparticle image velocimetry (micro-PIV) Meas. Sci. Technol. 20 doi:10.1088/0957-0233/20/6/065401

[12] Cerqueira R F L, Paladino E E, Ynumaru B K and Maliska C R 2018 Image processing techniques for the measurement of two-phase bubbly pipe flows using particle image and tracking velocimetry (PIV/PTV) Chem. Eng. Sci. 189 $1-23$

[13] Schubring D, Shedd T A and Hurlburt E T 2010 Planar laser-induced fluorescence (PLIF) measurements of liquid film thickness in annular flow. Part I: Methods and data Int. J. Multiph. Flow 36 815-824

[14] Schubring D, Shedd T A and Hurlburt E T 2010 Planar laser-induced fluorescence (PLIF) measurements of liquid film thickness in annular flow. Part II: Analysis and comparison to models Int. J. Multiph. Flow 36 825-835

[15] Cherdantsev A V, An J S, Charogiannis A and Markides C N 2019 Structured planar laser-induced fluorescence (SPLIF) for the accurate identification of interfaces in multiphase flows Int. J. Multiph. Flow 118 193-204

[16] Cherdantsev A V, An J S, Charogiannis A and Markides C N 2019 Simultaneous application of two laser-induced fluorescence approaches for film thickness measurements in annular gas-liquid flows Int. J. Multiph. Flow 119 237-258

[17] Murzyn F, Mouaze D and Chaplin J R 2007 Air-water interface dynamic and free surface features in hydraulic jumps J. Hydraul. Res. 45 679-685

[18] Murzyn F, Mouaze D and Chaplin J R 2005 Optical fibre probe measurements of bubbly flow in hydraulic jumps Int. $J$. Multiph. Flow 31 141-154

[19] Teng P and Yang J 2018 Modeling and Prototype Testing of Flows over Flip-Bucket Aerators J. Hydraul. Eng. 144 1-14 10.1061/(ASCE)HY.1943-7900.0001531

[20] Pfister M and Gisonni C 2014 Head Losses in Junction Manholes for Free Surface Flows in Circular Conduits J. Hydraul. Eng. 140 1-6 10.1061/(ASCE)HY.19437900.0000895

[21] Felder S and Chanson H 2018 Air-Water Flow Patterns of Hydraulic Jumps on Uniform Beds Macroroughness $J$. Hydraul. Eng. 144 10.1061/(ASCE)HY.1943-7900.0001402.

[22] Bung D B 2013 Non-intrusive detection of air-water surface roughness in self-aerated chute flows J. Hydraul. Res. $51322-329$

[23] Zhang G, Valero D, Bung D B and Chanson H 2018 On the estimation of free-surface turbulence using ultrasonic sensors Flow Meas. Instrum. 60 171-184
[24] Rak G, Steinman F, Hočevar M, Dular M, Jezeršek M and Pavločič U 2017 Laser ranging measurements of turbulent water surfaces $2^{\text {nd }}$ International Symposium on Image based Metrology, Maui, Hawaii

[25] Brudu N and Lague D 2012 3D terrestrial lidar data classification of complex natural scenes using a multi-scale dimensionality criterion: Applications in geomorphology ISPRS J. Photogramm. Remote Sens. 68 121-134

[26] Chen Q, Wang H, Zhang H C, Sun M W and Liu X G 2016 A Point Cloud Filtering Approach to Generating DTMs for Steep Mountainous Areas and Adjacent Residential Areas Remote Sens. 8 1-22

[27] Prufer K M, Thompson A E and Kennett D J 2015 Evaluating airborne LiDAR for detecting settlements and modified landscapes in disturbed tropical environments at Uxbenka Belize J. Archaeol. Sci. 57 1-13

[28] Smith M, Vericat D and Gibbins C 2012 Through-water terrestrial laser scanning of gravel beds at the patch scale. Earth Surf. Process. Landforms 37 411-421

[29] Tamari S, Mory J and Guerrero-Meza V 2011 Testing a nearinfrared LIDAR mounted with a large incidence angle to monitor the water level of turbid reservoirs ISPRS J Photogramm Remote Sens 66 85-91

[30] Allis M J, Peirson W L, Banner and M L 2011 Application of LIDAR as a measurement tool for waves Proceedings of the Twenty-first International Offshore and Polar Engineering Conf. 19-24

[31] Blenkingsopp C E, Turner I L, Allis M J, Peirson W L and Garden L E 2012 Application of LiDAR technology for measurement of time-varying free-surface profiles in a laboratory wave flume Coast. Eng. J. 68 1-5

[32] Blenkinsopp C E, Mole M A, Turner I L and Peirson W L 2010 Measurements of the time-varying free-surface profile across the swash zone obtained using an industrial LIDAR Coastal Eng. 57 1059-1065

[33] Martins K, Blenkinsopp C E, Power H E, Bruder B, Puleo J A and Bergsma E W 2017 High-resolution monitoring of wave transformation in the surf zone using a LiDAR scanner array Coast. Eng. 128 37-43

[34] Martins K, Blenkinsopp C E and Zang J 2016. Monitoring individual wave characteristics in the inner surf with a 2dimensional laser scanner (LiDAR) J. Sens. 2016 1-11

[35] Martins K, Bonneton P, Frappart F, Detandt G, Bonneton N and Blenkinsopp C E 2017 High frequency field measurements of an undular bore using a 2D LiDAR scanner. Remote Sens. 9 1-14

[36] Kramer M, Chanson H and Felder S 2019 Can We Improve the Non-Intrusive Characterisation of High-velocity Air-Water Flows? Application of Lidar Technology to Stepped Spillways. J. Hydraul. Res. 1-13

[37] Rak G, Hočevar M and Steinman F 2017 Measuring water surface topography using laser scanning Flow Meas. Instrum. $56,35-44$

[38] Rak G, Hočevar M and Steinman F 2019 Water surface topology of supercritical junction flow J. Hydrol. Hydromech. $67154-162$

[39] Montano L, Li R and Felder S. 2018 Continuous Measurements of Time-Varying Free-Surface Profiles in 
Aerated Hydraulic Jumps with a Lidar. Exp Therm Fluid Sci 93 379-397

[40] Montano L and Felder S 2018 LIDAR measurements of free-surface profiles and turbulent scales in a hydraulic jump 7th international symposium on hydraulic structures (ISHS), Aachen 